\title{
Bladder exstrophy-epispadias complex with adenocarcinoma in an adult patient: $A$ case report
}

\author{
WEI XIONG ${ }^{1}$, RAN PENG $^{2}$, LIANG ZHU ${ }^{1}$ and ZHAOHUI ZHONG ${ }^{1}$ \\ Departments of ${ }^{1}$ Urology and ${ }^{2}$ Cardiology, The Second Xiangya Hospital, \\ Central South University, Changsha, Hunan 410011, P.R. China
}

Received July 13, 2014; Accepted August 26, 2015

DOI: $10.3892 /$ etm.2015.2793

\begin{abstract}
The present case report presents the case of exstrophy-epispadias complex (EEC) with the widest separation of the symphysis pubis to date. A 33-year-old male was referred to hospital with previously unreconstructed EEC malformation. The diagnosis established was of EEC with separation of the symphysis pubis and left inguinal hernia with adenocarcinoma of the bladder. The patient underwent radical cystectomy plus an ileal conduit and an expanded local lymphadenectomy. Osteotomy was not performed, and instead the defect of the pelvis and abdominal wall was repaired using meshes and a lower abdominal island skin flap. At 1 year after surgery the patient recovered well, with no indications of mesh distortion or major complications.
\end{abstract}

\section{Introduction}

Bladder exstrophy is typically associated with a specific set of conditions, including epispadias, hernia and pelvic malformation. This spectrum of abnormalities is known as the exstrophy-epispadias complex (EEC) (1). It is estimated that EEC occurs in $\sim 1 / 35,000$ live births (2), and the risk of bladder carcinoma in the EEC population is 700-times higher compared with that of the age-matched general population (3). Ideally the management of EEC is initiated in the neonatal period, as malignant potential increases with age if the condition remains untreated (4). As the majority of patients with EEC are treated when they are infants, adult cases of EEC are rarely reported.

\section{Case report}

A 32-year-old man with bladder exstrophy was referred to the Second Xiangya Hospital of Central South University

Correspondence to: Professor Zhaohui Zhong, Department of Urology, The Second Xiangya Hospital, Central South University, 139 Renmin Road, Changsha, Hunan 410011, P.R. China

E-mail: jzhongguro@163.com

Key words: adenocarcinoma, adult, bladder exstrophy, epispadias
(Changsha, China) in February 2013, having received no previous surgical intervention. The patient complained of intermittent bladder pain and bleeding, and complete incontinence that required the continual use of diapers. Since adolescence, the patient's penis had exhibited a limited degree of development; however, it was able to enlarge and eject seminal fluid in response to sexual stimulation.

A physical examination revealed that the umbilicus was absent, replaced by a scarlet-colored bladder plate of $5 \times 6 \mathrm{~cm}$, which was partially thickened and exhibited mucosal erosion. The ureters opened at each side of the lower part of the bladder plate, and clear urine was release intermittently from each ureter. A $2-\mathrm{cm}$ long epispadias penis with a splayed glans was present beneath the bladder plate. The left testicle was impalpable and the right was normal. A number of painless enlarged lymph nodes were identified in the left inguinal area. The perineum was shortened as the anus was located immediately behind the scrotum. Numerous reddish broad-based neoplasms were identified on the bladder plate (Fig. 1).

Laboratory analysis, including routine blood tests, indicated no abnormalities in renal or liver function. X-ray examination showed a separation of the symphysis pubis of $\leq 102 \mathrm{~mm}$ (Fig. 2). Computed tomography scanning revealed that the bladder cavity was absent and the bladder plate was thickened and protruded, and a hernia protruding into the left scrotum chamber was confirmed. Magnetic resonance imaging showed no enhancement of the bladder plate and the signal of the prostate was homogenous, indicating no abnormality. No enlarged lymph nodes were observed in the pelvic cavity. Ultrasonography indicated normal bilateral kidneys and adrenal glands. A biopsy of the bladder plate neoplasms revealed severe atypical hyperplasia with well-differentiated adenocarcinoma. No other abnormalities or tumor sites were detected.

The patient underwent radical cystectomy and urinary diversion with a ileal conduit, and an expanded local lymphadenectomy was performed. Subsequently, a 10x8-cm gap was present in the lower abdomen. Osteotomy was not performed; instead a customized titanium mesh was applied to bridge the gap. The mesh was fixed to the pubic and ischial ramus, and an onlay mesh repair was performed to correct the hernia (Fig. 2).

A $12 \times 8-\mathrm{cm}$ lower abdominal island skin flap was specifically designed, the pedicle of which was located in the left inguinal region. The flap was rotated $\sim 45^{\circ}$ to cover the skin of the defective area. 


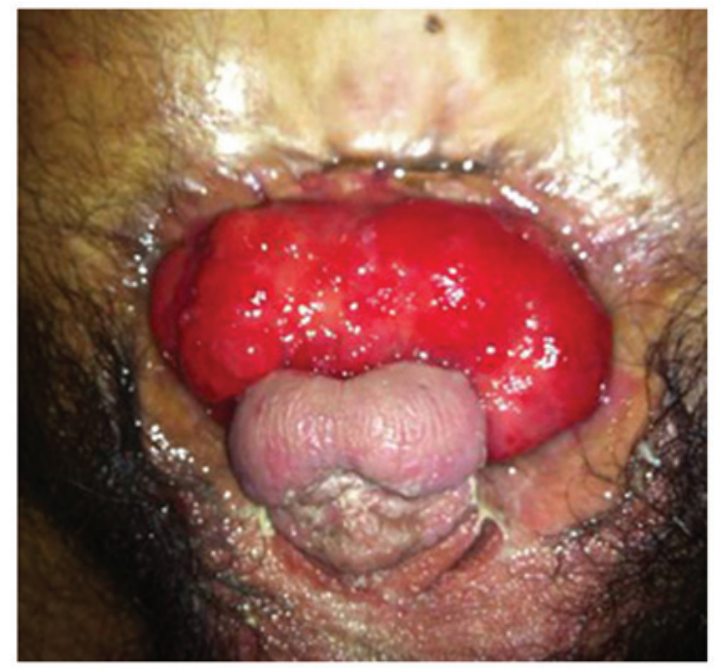

Figure 1. Pre-surgery view of the patient with exstrophy-epispadias complex. Bladder plate showing reddish, broad-based neoplasms.
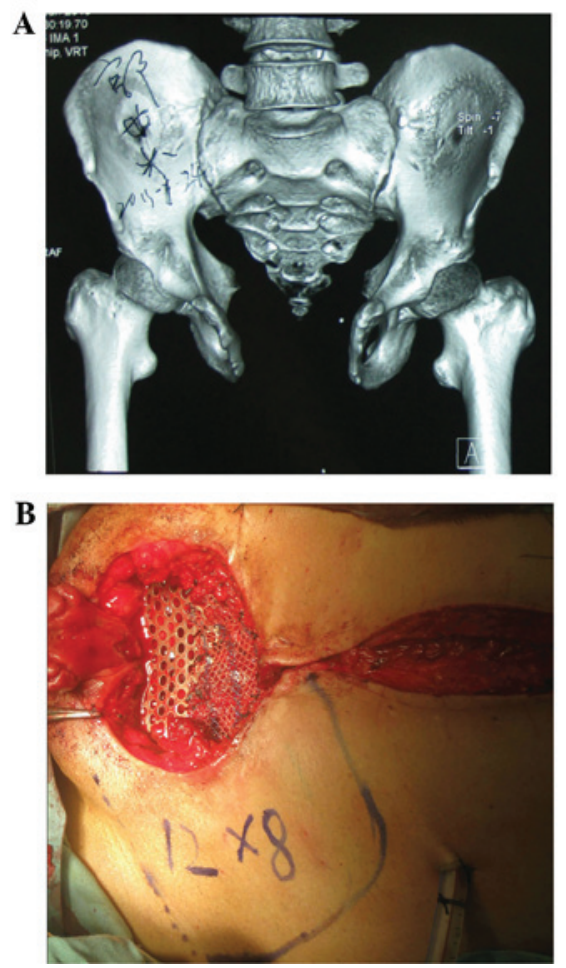

Figure 2. (A) Computed tomography three-dimensional reconstruction of the pelvis; the separation of the symphysis pubis was $\leq 102 \mathrm{~mm}$. (B) Intraoperative view of the patient with the metallic mesh installed, and the specifically designed skin flap depicted.

The reconstruction of the penis was not conducted, but postponed until following the successful completion of the current surgery.

Postoperative pathological examination revealed that the bladder tumor was a well-differentiated adenocarcinoma. No cancer tissue was detected in the resected prostate or lymph nodes. Following surgery, no adjuvant therapy was applied. At a 1-year follow-up examination, the patient was recovering well, claiming to experience a much better quality of life. The patient expressed no intention to undergo reconstruction

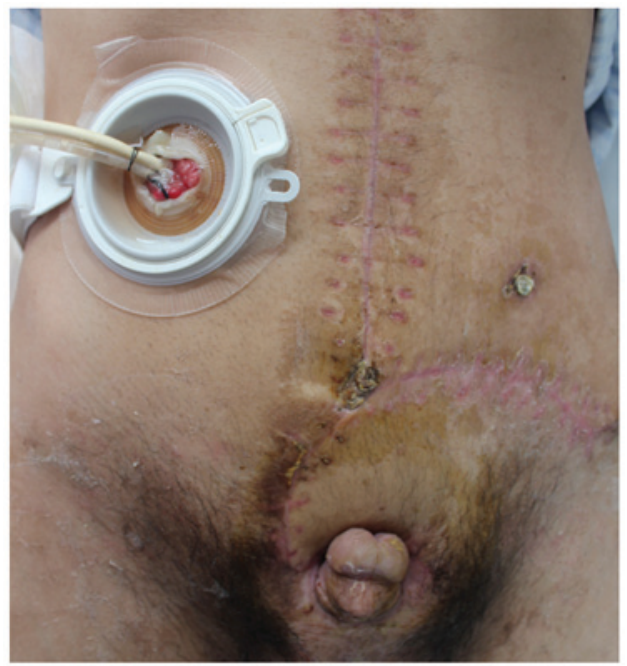

Figure 3. Image at 1 month after surgery. The patient recovered well. Reconstruction of the penis was postponed until following the present surgery.

of the penis (Fig. 3). This study was approved by the ethics committee of the Second Xiangya Hospital of Central South University. Informed consent was obtained from the patient prior to their contribution to this study.

\section{Discussion}

In cases of EEC in adult patients, careful screening for malignant lesions in the affected areas is necessary. Notably, the majority of untreated cases of EEC with bladder cancer have been identified as adenocarcinoma or squamous cell carcinoma, which are rare histological types of primary bladder cancer $(5,6,7)$. The usual primary sites of these types include the gastrointestinal system, breasts, prostate and ovaries (5).

As in numerous other complicated conditions, it is necessary for the treatment for adult EEC to be designed according to the circumstances of the individual patient. In the present case, the aims of the surgery included: i) Controlling the malignant lesions; ii) improving the quality of life of the patient; and iii) improving the patient's cosmetic appearance.

Therefore, a radical cystectomy plus expanded local lymphadenectomy was performed. In addition, an ileal conduit was applied as the patient had no normal urethra.

The performance of an osteotomy in exstrophy reconstruction is controversial. Proponents of the use of osteotomy maintain that it facilitates easier approximation of the soft tissues; the opponents are primarily concerned about the risk of inadvertently causing future incontinence (8). To the best of our knowledge, the present patient had the widest separation of the symphysis pubis reported in the literature to date. Considering the width of the gap and the rigidity of the pelvic bone, there was risk of an osteotomy reducing the stability of the patient's pelvis. Therefore, an osteotomy was not performed. Instead, a metallic mesh was used to restore the integrity of the pelvis and to help reconstruct the lower abdomen wall. On the basis of multiple follow-up examinations, the mesh appeared to function well for the patient. At a 1-year follow-up examination, neither mesh distortion nor other complications were detected. 


\section{Acknowledgements}

The authors thank the anesthesiologists, nurses, care-givers and hospital workers who facilitated the surgery.

\section{References}

1. Gearhart JP: The bladder exstrophy-epispadias-cloacal exstrophy complex. In: Pediatric Urology. Volume. Gearhart JP, Rink RC and Mouriquand PDE (eds). Saunders, Philadelphia, pp511-546, 2001.

2. Grossfeld GD and Carroll PR: Evaluation of asymptomatic microscopic hematuria. Urol Clin North Am 25: 661-676, 1998.

3. Smeulders N and Woodhouse CR: Neoplasia in adult exstrophy patients. BJU Int 87: 623-628, 2001.
4. Gulati P, Yadav SP and Sharma U: Management of bladder exstrophy in adulthood: Report of 2 cases. J Urol 157: 947-948, 1997.

5. Di Lauro G, Iacono F, Ruffo A, Romis L, Mordente S, Pane U, Illiano E, Romeo G, Prezioso D and Amato B: Presenting a case of a mucinous adenocarcinoma of an exstrophic bladder in an adult patient and a review of literature. BMC Surg 13 (Suppl 2): S36, 2013.

6. Rieder JM, Parsons JK, Gearhart JP and Schoenberg M: Primary squamous cell carcinoma in unreconstructed exstrophic bladder. Urology 67: 199, 2006.

7. Paulhac P, Maisonnette F, Bourg S, Dumas JP and Colombeau P: Adenocarcinoma in the exstrophic bladder. Urology 54: 744 1999.

8. Borer JG: Are osteotomies necessary for bladder exstrophy closure? J Urol 191: 13-14, 2014. 